\title{
AVALIAÇÃO FENOTÍPICA DE CARACTERÍSTICAS DE FRUTOS EM DUAS FAMÍLIAS DE MEIOS-IRMÃOS DE GOIABEIRA-SERRANA (Acca sellowiana Berg.) DE UM POMAR COMERCIAL EM SÃO JOAQUIM, SC ${ }^{1}$
}

\author{
JULIANA DEGENHARDT²; JEAN-PIERRE DUCROQUET ${ }^{3}$; MIGUEL PEDRO GUERRA ${ }^{4}$; RUBENS ONOFRE NODARI ${ }^{4}$
}

RESUMO - Frutos de um pomar comercial composto por progênies de duas plantas matrizes foram avaliados, com o objetivo de caracterizar a variabilidade fenotípica. As características peso de fruto, diâmetro e sólidos solúveis totais apresentaram diferenças estatísticas significativas entre as médias de famílias em pelo menos dois dos anos avaliados, enquanto para comprimento e rendimento de polpa a diferença não foi significativa. Houve diferença significativa entre médias de anos, dentro de cada família, para todas as características, com exceção das médias de peso de fruto entre 1998 e 1999 nas duas famílias e as médias de sólidos solúveis totais entre 1998 e 1999 em uma família e entre 1999 e 2000 na outra família. As correlações e regressões entre características produtivas mais relevantes foram obtidas entre peso de fruto e peso de casca, peso de fruto e comprimento, peso de fruto e diâmetro, peso de casca e comprimento, peso de casca e diâmetro e comprimento e diâmetro.

Termos para indexação: variabilidade, correlação, regressão

\section{PHENOTYPIC EVALUATION OF FRUIT TRAITS OF TWO HALF SIB FAMILIES OF FEIJOA (Acca sellowiana Berg.) FROM AN ORCHARD IN SÃO JOAQUIM, SANTA CATARINA}

\begin{abstract}
Fruits of a commercial orchard originated by seedlings from two plants were evaluated, with the objective to characterize the phenotypic variation. Statistical differences were found among family means in at least two years for fruit diameter and solids soluble contents, but not for fruit length and pulp revenue. There were statistically significant differences in means among year means, within each family, for all the fruits traits, except for fruit weight between 1998 and 1999 in both families, and for soluble solids contents between 1998 and 1999 for family 1 and between 1999 and 2000 for the other family. The most important correlations and regressions among productive traits were obtained among fruit weight and peel weight, fruit weight and length, fruit weight and diameter, peel weight and length, peel weight and diameter and length and diameter.

Index terms: variability, correlation, regression
\end{abstract}

\section{INTRODUÇÃO}

A Acca sellowiana é uma mirtácea originária do planalto meridional brasileiro (Mattos, 1986). A espécie é cultivada no Brasil, em sua maioria, em caráter doméstico e extrativo. Seus frutos mostram, no entanto, grande potencial econômico, devido a suas características organolépticas, sendo empregados na fabricação de sucos, geléias, sorvetes e licores, entre outros (Sharpe et al.,1993; Ducroquet et al., 2000). Na Nova Zelândia, já são 13 produtos feitos a partir do fruto (Thorp \& Bieleski, 2002).

Os frutos da espécie, classificados como um pseudo-fruto do tipo pomo, são semelhantes à goiaba comum em aparência, tamanho e textura, mas a polpa, de cor gelo, apresenta sabor e aroma doceacidulados, bastante distintos (Ducroquet \& Ribeiro, 1991). O peso pode variar de menos de 30 a mais de $250 \mathrm{~g}$ e o rendimento de polpa atinge geralmente valores máximos de 50\% (Ducroquet et al., 2000). A casca pode apresentar aspecto liso, rugoso ou semi-rugoso (Mattos, 1986).

Apesar de não apresentar cultivo expressivo no Brasil, a goiabeira-serrana já é comercializada nos Estados Unidos e Europa, a partir de cultivares desenvolvidas principalmente na Nova Zelândia e Estados Unidos, para onde a espécie foi levada no início do século passado (Ducroquet et al., 2000). A avaliação de sua variabilidade fenotípica é ainda incipiente no Brasil, seu centro de origem, uma vez que a espécie não é considerada domesticada. Os poucos pomares comerciais, além de plantas isoladas nos quintais de residências na região de ocorrência natural, são oriundos de mudas feitas a partir de sementes, pois não há ainda no país variedades melhoradas.

Esse estudo teve por objetivo avaliar a variação fenotípica de características de frutos de plantas de duas famílias de meios irmãos e estimar os coeficientes de correlação e as equações de regressão entre características produtivas e entre características produtivas e vegetativas. Assim, este trabalho é pioneiro na avaliação da amplitude de variação destas características em pomar comercial, como também avançar no conhecimento que pode auxiliar a melhoria da eficiência dos programas de melhoramento, que buscam o desenvolvimento de cultivares para plantios comerciais.

\section{MATERIALE MÉTODOS}

O pomar avaliado localiza-se no Município de São Joaquim, SC, a $1.350 \mathrm{~m}$ de altitude. Implantado em 1985, é composto por 889 plantas. As plantas foram todas obtidas a partir de sementes, sendo as filas 1 a 17 progênies de uma planta matriz (FMI1) e as filas 18 a 26 progênies de outra planta matriz (FMI2). As duas plantas matrizes foram escolhidas no campo, por apresentarem características de fruto e arquitetura de plantas consideradas desejáveis pelo produtor.

As amostras, que consistiram de 5 a 10 frutos maduros por planta, foram coletadas em março e abril dos anos de 1998, 1999 e 2000. Após a coleta, os mesmos foram armazenados à temperatura de $7^{\circ} \mathrm{C}$ até sua utilização. No ano de 1998, foram coletados frutos de 186 plantas do pomar, 87 da FMI1 e 99 da FMI2, tomadas ao acaso entre as plantas com frutificação. Frutos de uma sub-amostra de 90 plantas escolhidas ao acaso, dentre as 186 amostradas em 1998, sendo 46 da FMI1 e 44 FMI2, foram também coletados nos anos de 1999 e 2000. Nos três anos, as características avaliadas foram: peso de fruto $(\mathrm{PF})$; peso de casca (PC); rendimento de polpa (RP); comprimento (CO); diâmetro (DI); relação comprimento/diâmetro $(\mathrm{C} / \mathrm{D})$, cujos valores foram classificados como: Classe 1 - $<1,1$; Classe 2 - entre 1,11 e 1,3; Classe 3 - entre 1,31 e 1,5 e Classe $4->1,5$; sólidos solúveis totais (SST), medido com refratômetro e expresso em porcentagem e tipo de casca (rugoso, semirugoso ou liso).

Foi realizada a análise descritiva (média, desvio-padrão, coeficiente de variação e valores máximos e mínimos) das características nos três anos de avaliação. As duas famílias foram comparadas entre si e entre os anos, com base no t-teste. Posteriormente, foram estimados

${ }^{1}$ (Trabalho 191/2002). Recebido: 18/10/2002; Aceito para publicação: 24/10/2003

${ }_{2}^{2}$ Mestre em Recursos Genéticos Vegetais, CCA/UFSC; E-mail: julianadt@hotmail.com.

${ }^{3}$ Doutor em Biologia e Fisiologia Vegetal, Pesquisador da EPAGRI, São Joaquim, SC.

${ }^{4}$ Professores Doutores do CPG - Recursos Genéticos Vegetais - CCA/UFSC; Depto de Fitotecnia; CP-476, Florianópolis, SC; CEP 88040-900. E-mail: nodari@mbox1.ufsc.br 
os coeficientes de correlação de Pearson e as análises de regressão entre as características de produção. Para tal, foram utilizados os dados primários bem como os dados da relação comprimento/diâmetro (C/D).

\section{RESULTADOS E DISCUSSÃO}

De uma maneira geral, as famílias de meios irmãos quanto às plantas dentro das famílias mostraram variação considerável entre si para todas as características (Tabela 1). Os coeficientes de variação indicam haver maior variabilidade entre as plantas para PF e RP. Esses valores sugerem que existe espaço para a seleção nessas características, uma vez que a variação entre indivíduos é bastante expressiva.

Não houve diferenças estatísticas entre famílias para RP e CO em nenhum dos anos avaliados, demostrando que a seleção baseada nessas características não priorizaria uma família em particular (Tabela 1). Para as demais características, entretanto, houve diferenças estatísticas em pelo menos dois dos anos avaliados. Se a seleção de frutos grandes fosse desejada, essa priorizaria as plantas da FMI2.

A diferença entre as médias de características entre os anos, para cada família separadamente, também foi avaliada (Tabela 1). Os resultados mostraram a importância do efeito de anos e a grande variação ambiental sofrida pelas mesmas, sugerindo baixos valores de herdabilidade. A partir desses resultados, verificou-se que o PF foi estatisticamente superior no terceiro ano e o RP foi estatisticamente inferior. Apesar disso observou-se nos genótipos estudados, plantas com frutos de maior peso e maior rendimento de polpa, em sua maioria entre as plantas da FMI2. Esse fato já foi observado em Jabuticabeira, para a qual frutos de maior peso apresentaram menor rendimento. Também nesse caso, dentre o material avaliado, foi possível a identificação de plantas com frutos grandes e maior rendimento de polpa (Pereira et al., 2000).

$\mathrm{O}$ CO e o DI foram estatisticamente superiores no terceiro ano de avaliação, sugerindo que o aumento destas características contribuem de maneira semelhante para o aumento do tamanho dos frutos, não havendo, portanto, grande variação no formato dos frutos, o que ficou comprovado pela baixa variação na relação C/D entre os anos. $\mathrm{O}$ formato de frutos também foi variável em estudo de Jabuticabeiras e Goibeiras (Manica et al., 2000) (ambas da família Myrtaceae). Na primeira, nenhuma das plantas avaliadas caracterizou-se por produzir fruto de determinado formato (Pereira et al., 2000).

A variação entre anos para SST não ocorreu de maneira semelhante entre as duas famílias (Tabela 1). Isso sugere que a diferença no clima e temperatura entre os anos não foram capazes de afetar sobremaneira essa característica. Os valores de SST foram variáveis entre as plantas (Tabela 1). Entretanto, há que se considerar que o valor de SST não deve ser selecionado por si só, uma vez que a relação entre esta característica e a acidez titulável é que determina o sabor dos frutos.

Os três tipos de casca ocorreram entre as plantas das duas famílias, mostrando a grande variabilidade dentro de progênies. Além disso, as plantas não necessariamente apresentaram o mesmo tipo nos três anos de avaliação. Algumas plantas que tiveram frutos classificados como lisos ou rugosos em um ano, tiveram seus frutos classificados como semi-rugosos em outro ano e vice-versa. No entanto, apesar da possível confusão entre as duas classes com o tipo semi-rugoso, frutos lisos nunca foram confundidos com frutos rugosos, devido à grande diferença entre essas classes. Em todos os anos, a maioria das plantas avaliadas apresentou frutos com casca semi-rugosa e entre as plantas da FMI1, plantas com frutos rugosos representaram sempre menos de $10 \%$ da população.

As correlações mais relevantes foram encontradas entre PF e $\mathrm{PC}, \mathrm{PF}$ e CO, PF e DI, PC e CO e PC e DI, todas de magnitude variando de 0,66 a 0,98 e muito semelhantes entre os anos de avaliação e entre as famílias. Isto sugere que de $40 \%$ a $96 \%$ da variação é comum as duas caracteríscticas consideradas. Assim, quanto maior o peso de fruto, maior será o peso da casca, o comprimento e o diâmetro do fruto. Por outro lado, os valores dos coeficientes de correlações negativos foram baixos, exceção aqueles envolvendo RP (Tabela 2 ). Se negativos estes coeficientes indicam que quando o valor de uma característica aumenta, o valor da outra diminui. Contudo, na maioria dos casos estes valores foram baixos, indicando que a associação é muito fraca.

TABELA 1 - Médias, valores máximos e mínimos e coeficientes de variação para as características peso de fruto, rendimento de polpa, comprimento, diâmetro, relação comprimento/diâmetro e sólidos solúveis totais para a FMI1 e a FMI2 nos anos de 1998, 1999 e 2000. São Joaquim, SC.

\begin{tabular}{|c|c|c|c|c|c|c|c|c|}
\hline 1998 & \multicolumn{4}{|c|}{ Progênies PMI1 $(n=66)$} & \multicolumn{4}{|c|}{ Progênies PMI2 (n=120) } \\
\hline Característica & Média & CV (\%) & Máx & Mín & Média & CV (\%) & Máx & Mín \\
\hline Peso de fruto $(\mathrm{g})$ & $62,2 \mathrm{a}$ & 31,7 & 95,7 & 30,9 & $69,5 \mathrm{a}$ & 31,9 & 116,6 & 43,6 \\
\hline Rendimento de polpa (\%) & $33,7 \mathrm{~A}$ & 20,3 & 47,4 & 20,6 & $32,9 \mathrm{~A}$ & 21,8 & 47,8 & 20,0 \\
\hline Comprimento $(\mathrm{cm})(\mathrm{C})$ & $5,4 \mathrm{~A}$ & 13,7 & 6,8 & 3,8 & $5,6 \mathrm{~A}$ & 15,8 & 7,6 & 4,4 \\
\hline Diâmetro (cm) (D) & 4,2 & 8,4 & 5,0 & 3,3 & 4,5 & 11,3 & 5,6 & 3,6 \\
\hline $\mathrm{C} / \mathrm{D}$ & 1,2 & 13,1 & 1,6 & 1,0 & 1,2 & 12,6 & 1,6 & 1,0 \\
\hline SST $(\%)$ & $10,5 \mathrm{a}$ & 16,5 & 14,3 & 7,3 & 9,7 & 16,5 & 12,8 & 7,1 \\
\hline 1999 & \multicolumn{4}{|c|}{ Progênies PMI1 (n=46) } & \multicolumn{4}{|c|}{ Progênies PMI2 (n=44) } \\
\hline Característica & Média & CV (\%) & Máx & Mín & Média & CV (\%) & Máx & Mín \\
\hline Peso de fruto $(\mathrm{g})$ & $64,4 \mathrm{aA}$ & 35,2 & 126,0 & 30,0 & $67,5 \mathrm{aA}$ & 31,2 & 98,2 & 26,7 \\
\hline Rendimento de polpa (\%) & $28,7 \mathrm{~A}$ & 26,0 & 45,9 & 16,6 & $28,8 \mathrm{~A}$ & 27,9 & 43,9 & 15,6 \\
\hline Comprimento (cm) (C) & $4,8 \mathrm{~A}$ & 19,1 & 6,8 & 3,2 & $4,8 \mathrm{~A}$ & 16,2 & 5,8 & 3,2 \\
\hline Diâmetro (cm) (D) & $4,0 \mathrm{~A}$ & 13,9 & 5,4 & 3,0 & $4,1 \mathrm{~A}$ & 12,7 & 5,1 & 2,9 \\
\hline Comp/Diam & 1,2 & 15,6 & 1,7 & 1,0 & 1,2 & 12,6 & 1,5 & 0,9 \\
\hline SST $(\%)$ & $10,5 \mathrm{a}$ & 13,2 & 12,6 & 8,3 & $11,0 \mathrm{a}$ & 12,1 & 12,8 & 8,9 \\
\hline 2000 & \multicolumn{4}{|c|}{ Progênies PM1 (n=46) } & \multicolumn{4}{|c|}{ Progênies PM2 $(n=44)$} \\
\hline Característica & Média & CV (\%) & Máx & Mín & Média & CV (\%) & Máx & Mín \\
\hline Peso de fruto $(\mathrm{g})$ & 81,1 & 37,2 & 165,2 & 41,2 & 91,0 & 33,9 & 152,6 & 52,7 \\
\hline Rendimento de polpa (\%) & $24,2 \mathrm{~A}$ & 36,4 & 35,3 & 13,2 & $23,5 \mathrm{~A}$ & 48,5 & 44,9 & 12,3 \\
\hline Comprimento $(\mathrm{cm})(\mathrm{C})$ & $6,4 \mathrm{~A}$ & 16,7 & 8,6 & 4,7 & $6,6 \mathrm{~A}$ & 15,8 & 8,6 & 4,7 \\
\hline Diâmetro (cm) (D) & 4,8 & 13 & 6,3 & 3,9 & 5,1 & 12,3 & 6,1 & 4 \\
\hline $\mathrm{C} / \mathrm{D}$ & 1,3 & 18 & 1,8 & 1,0 & 1,3 & 15,5 & 1,8 & 1,0 \\
\hline SST $(\%)$ & $11,0 \mathrm{~A}$ & 12,5 & 12,7 & 9,0 & $10,9 \mathrm{aA}$ & 12,1 & 12,8 & 9,1 \\
\hline
\end{tabular}

Médias das características de anos diferentes mas da mesma família seguidas da mesma letra minúscula (análise vertical) e médias dentro do mesmo ano mas de famílias diferentes seguidas da mesma letra maiúscula (análise horizontal) não são diferentes estatisticamente pelo t-teste (5\% de probabilidade). 
TABELA 2 - Coeficientes de correlação entre as características peso de fruto (PF), peso de casca (PC), rendimento de polpa (RP), comprimento (CO), diâmetro (DI), sólidos solúveis totais (SST) avaliados em plantas de goiabeira serrana nos anos de 1998, 1999 e 2000. Os valores acima da diagonal são referentes às correlações entre as características para as PM1 e os valores abaixo da diagonal para as PM2. São Joaquim, SC.

\begin{tabular}{|c|c|c|c|c|c|c|c|}
\hline \multicolumn{2}{|l|}{1998} & \multicolumn{6}{|c|}{ FMI1 } \\
\hline \multirow{7}{*}{ FMI2 } & & PF & $\mathrm{PC}$ & RP & $\mathrm{CO}$ & DI & SST \\
\hline & PF & 1 & $0,96^{* *}$ & $-0,06^{\mathrm{NS}}$ & $0,86^{* *}$ & $0,93 * *$ & $-0,14^{* *}$ \\
\hline & $\mathrm{PC}$ & $0,94 * *$ & 1 & $-0,32 * *$ & $0,83 * *$ & $0,87 * *$ & $-0,07^{\mathrm{NS}}$ \\
\hline & $\mathrm{RP}$ & $0,33 * *$ & $-0,31 * *$ & 1 & $-0,04^{\mathrm{NS}}$ & $0,05^{\mathrm{NS}}$ & $-0,24 * *$ \\
\hline & $\mathrm{CO}$ & $0,81 * *$ & $0,74 * *$ & $0,13 * *$ & 1 & $0,68 * *$ & $-0,13^{* *}$ \\
\hline & DI & $0,90 * *$ & $0,88^{* *}$ & $-0,09 * *$ & $0,52 * *$ & 1 & $-0,25^{* *}$ \\
\hline & SST & $-0,17 * *$ & $-0,24 * *$ & $0,23 * *$ & $-0,19 * *$ & $-0,17 * *$ & 1 \\
\hline \multirow[t]{4}{*}{1999} & & \multicolumn{6}{|c|}{ FMI1 } \\
\hline & & $\mathrm{PF}$ & PC & $\mathrm{RP}$ & $\mathrm{CO}$ & DI & SST \\
\hline & $\mathrm{PF}$ & 1 & $0,98 * *$ & $-0,36^{* *}$ & $0,73 * *$ & $0,93 * *$ & $0,27 * *$ \\
\hline & $\mathrm{PC}$ & $0,90 * *$ & 1 & $-0,53 * *$ & $0,76 * *$ & $0,91 * *$ & $0,23 * *$ \\
\hline \multirow[t]{4}{*}{ FMI2 } & $\mathrm{RP}$ & $0,04^{\mathrm{NS}}$ & $-0,38 * *$ & 1 & $-0,33 * *$ & $-0,36 * *$ & $0,06^{\mathrm{NS}}$ \\
\hline & $\mathrm{CO}$ & $0,77 * *$ & $0,75 * *$ & $-0,10^{\mathrm{NS}}$ & 1 & $0,48 * *$ & $0,21 * *$ \\
\hline & DI & $0,90 * *$ & $0,83 * *$ & 0,00 & $0,49 * *$ & 1 & $0,25 * *$ \\
\hline & SST & $-0,03^{\mathrm{NS}}$ & $0,10^{\mathrm{NS}}$ & $-0,33 * *$ & $-0,02^{\mathrm{NS}}$ & 0,00 & 1 \\
\hline \multirow[t]{4}{*}{2000} & & \multicolumn{6}{|c|}{ FMI1 } \\
\hline & & PF & PC & $\mathrm{RP}$ & $\mathrm{CO}$ & DI & SST \\
\hline & PF & 1 & $0,98 * *$ & $-0,44 * *$ & $0,66^{* *}$ & $0,89 * *$ & $0,25 * *$ \\
\hline & PC & $0,95 * *$ & 1 & $-0,59 * *$ & $0,66^{* *}$ & $0,87 * *$ & $0,30 * *$ \\
\hline \multirow[t]{4}{*}{ FMI2 } & $\mathrm{RP}$ & $-0,11 * *$ & $-0,41 * *$ & 1 & $-0,40 * *$ & $-0,36 * *$ & $-0,34 * *$ \\
\hline & $\mathrm{CO}$ & $0,75 * *$ & $0,75^{* *}$ & $-0,20 * *$ & 1 & $0,32 * *$ & $0,10^{* *}$ \\
\hline & DI & 0,80 ** & $0,82 * *$ & $-0,07^{\mathrm{NS}}$ & $0,43 * *$ & 1 & $0,26^{* *}$ \\
\hline & SST & $-0,07^{\mathrm{NS}}$ & $0,02^{\mathrm{NS}}$ & $-0,28 * *$ & $-0,01^{\mathrm{NS}}$ & $-0,13^{* *}$ & 1 \\
\hline
\end{tabular}

** - Significativo ao nível de 5\% de probabilidade; NS - Não significativo.

As outras correlações, ainda que significativas, foram de magnitudes pouco consideráveis e mostraram variação entre anos e entre as famílias, sugerindo que foram afetadas em grande parte pelo ambiente. Tomando-se como exemplo, os coeficientes entre SST foram negativos com as demais características em 1998 e positivos em 1999, sugerindo que o controle genético desta característica é independente das demais. Vale destacar também a correlação entre PF e RP na FMI1, a qual não foi diferente de zero no primeiro ano, porém alcançou valores $\mathrm{de}-0,36 \mathrm{e}-0,44$ nos segundo e terceiro anos, quando o $\mathrm{PF}$ foi maior. $\mathrm{Na}$ PMI2, os coeficientes de correlação foram $0,33,0,04$ e $-0,11$, sem consistência ao longo dos anos. Assim, o melhoramento para rendimento em polpa é complexo, pois está moderadamente correlacionado negativamente com peso de frutos e peso da casca na PMI2 e não correlacionado com estas mesmas características na PMI1.

Também em Mangabeiras, verificou-se que os frutos pequenos apresentaram rendimento de polpa 3,16\% superior aos frutos grandes (Vieira Neto, 1997). Correlação negativa entre rendimento de polpa e peso de frutos também foi observada em Aceroleira, em estudo baseado em 14 clones (Carpentieri-Pípolo et al., 2000). Já o estudo da mesma espécie realizado em plantas de um pomar comercial, revelou a existência de correlação positiva, ainda que de baixo valor (Gomes et al., 2000), demostrando que pode ocorrer variação dentro de espécie. A existência dessa variação permite a seleção de plantas com correlação positiva. No caso das plantas de goiabeira-serrana avaliadas, os valores mais baixos de correlação encontrados na FMI2 e os valores positivos encontrados no primeiro ano sugerem uma maior possibilidade de selecionar entre essas plantas, genótipos com maior PF e RP.

A correlação entre PC e RP na FMI1 foi sempre negativa. Assim, a medida que aumenta o peso da casca diminui a quantidade de polpa, possivelmente devido à competição por demanda de metabólitos. Os valores foram maiores nos anos em que o $\mathrm{PF}$ foi maior, o que sugere que o aumento no peso médio de frutos é acompanhado por um maior aumento no peso de casca do que na quantidade de polpa produzida. $\mathrm{O}$ mesmo ocorreu entre na FMI2, porém a diferença na magnitude entre os três anos foi menor, revelando uma menor sensibilidade a variações de anos.

Os coeficientes de correlação obtidos entre SST e RP sugerem que o aumento no RP está relacionado, ainda que fracamente, a uma diminuição no conteúdo de SST, principalmente quando o peso médio dos frutos é maior. Correlação negativa entre estas duas características também foi observada em Aceroleira (Gomes et al., 2000).

$\mathrm{Na}$ Tabela 3 estão as equações de regressão linear, que apresentaram valores de coeficientes de regressão estatisticamente significativos, obtidas entre as características avaliadas. Considerandose as equações de regressão e também os coeficientes de detrminação, as associações mais estreitas foram aquelas obtidas entre PF e PC, PF e $\mathrm{CO}, \mathrm{PF}$ e DI, PC e CO, PC e DI e CO e DI. Do ponto de vista biológico significa que no primeiro ano de avaliação, para cada $1 \mathrm{~g}$ de aumento no PC, houve um aumento de 1,28 g no PF, em ambas as famílias. Esse aumento foi um pouco menor nos outros dois anos de avaliação. As equações demonstraram ainda que o aumento do PF causa uma variação semelhante no DI e no CO dos mesmos.

Não é possível sugerir o uso de seleção indireta de características importantes, como RP ou conteúdo de SST, a partir da seleção de características como PF, CO ou DI, de mais fácil mensuração, uma vez que as correlações foram baixas e variáveis. As correlações e regressões entre caracteres vegetativos e de produção não foram significativas (Tabelas 2 e 3 ).

As análises feitas neste trabalho indicam a possibilidade de utilização de alguns clones (genótipos) deste pomar comercial avaliado como progenitores de cruzamentos em programas de melhoramento da espécie. É necessário agora um estudo mais aprofundado a respeito da herança dessas características, para que se possa prever com segurança os progressos que podem ser obtidos com a seleção a partir de cruzamentos, uma vez que se dispõe de material promissor, cujas características poderiam ser combinadas em uma ou mais cultivares. 
TABELA 3 -Equações de regressão com valores de coeficientes significativos $(\mathrm{P}<0,05)$, para as características peso de fruto (PF), peso de casca (PC), rendimento de polpa (RP), comprimento (CO), diâmetro (DI), relação entre comprimento e diâmetro (C/D), sólidos solúveis totais (SST) avaliados em plantas de goiabeira serrana nos anos de 1998, 1999 e 2000. São Joaquim, SC.

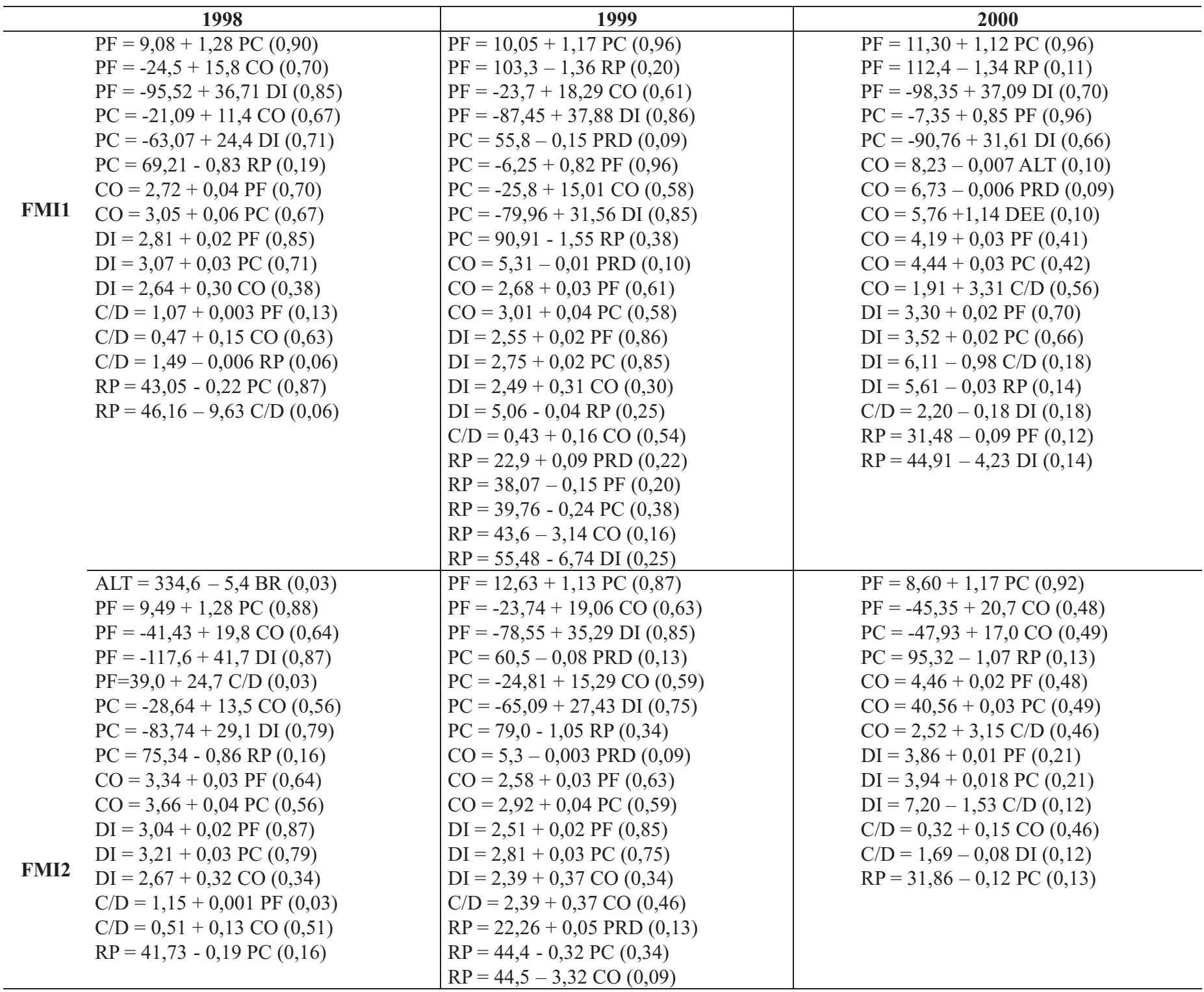

Valores entre parênteses correspondem aos coeficientes de determinação obtidos.

\section{CONCLUSÕES}

1) Houve variação fenotípica significativa entre as famílias para as características peso de fruto, diâmetro e sólidos solúveis totais em pelo menos dois dos anos avaliados;

2) As médias das características de frutos de cada família variaram entre anos, sendo altamente influenciadas pelo ambiente;

3) As correlações e regressões mais relevantes envonvendo peso de fruto sugerem que as características comprimento e diâmetro do fruto poderiam ser selecionadas simultaneamente;

4) Com base nas características de fruto, seria possível selecionar plantas deste pomar com características desejáveis para serem utilizadas em programas de melhoramento como progenitores de cruzamentos ou mesmo para propagação imediata, sendo que as progênies da segunda família, demonstraram, em geral, superioridade em características de importância agronômica.

\section{AGRADECIMENTOS}

Os autores agradecem ao Sr. Shu Otani, proprietário do pomar, o apoio financeiro do PRODETAB ao projeto, às agências CAPES pela bolsa a J.D. e ao CNPq pelas bolsas aos demais autores e aos revisores pela contribuição à qualidade deste artigo.

\section{REFERÊNCIAS BIBLIOGRÁFICAS}

CARPENTIERI-PÍPOLO,V., DESTRO, D., PRETE, C.E.C., GONZALES, M.G.N., POPPER, I., ZANATTA, S., SILVA, F.A.M. Seleção de genótipos parentais de acerola com base na divergência genética multivariada.Pesquisa Agropecuária Brasileira, Brasília, v.35, n.8, p.1613-1619,2000.

DUCROQUET, J.P.H.J.; RIBEIRO, P.A goiabeira serrana: velha conhecida, nova alternativa; Agropecuária Catarinense, Florianópolis, v.4, n.3, p.27-29, 1991.

DUCROQUET, J.P.H.J.; HICKEL, E.R.; NODARI, R.O. Goiabeira serrana (Feijoa sellowiana). Série Frutas Nativas 5; Jaboticabal: Funep, 2000, 66p.

GOMES, J.E., PERECIN, D.; MARTINS, A.B.G.; ALMEIDA, E.J. Variabilidade fenotípica em genótipos de acerola. Pesquisa Agropecuária Brasileira, Brasília, v.35, n.11, p.2205-2211, 2000. 
MANICA, I.; ICUMA, I.M.; JUNQUEIRA, N.T.V.; SALVADOR, J.O.; MOREIRA, A.; MALAVOLTA, E. Fruticultura tropical 6: Goiaba. Porto Alegre: Cinco Continentes, 2000, 374p.

MATTOS, J.R. A goiabeira serrana.Porto Alegre; Instituto de Pesquisas de Recursos Naturais Renováveis, Publicação IPRNR 19, 1986, 84p.

PEREIRA, M.C.T.; SALOMÃO, L.C.C.; MOTA, W.F.; VIEIRA, G. Atributos físicos e químicos de frutos de oito clones de jabuticabeiras. Revista Brasileira de Fruticultura, Jaboticabal, v.22, n. Especial, p.16-21, 2000
SHARPE, R. H.; SHERMAN, W. B.; MILLER, E. P. Feijoa history and improvement. Proceedings of the Florida State Horticultural Society, WinterHaven, v.106, p.134-139, 1993.

THORP, G.; BIELESKI, R. Feijoas: origins, cultivation and uses. Auckland: David Bateman, 2002. 87p.

VIEIRA NETO, R.D.. Caracterização física de frutos de uma população de mangabeiras (Hancornia speciosa Gomes). Revista Brasileira de Fruticultura, Cruz das Almas, v.19, n.2, p.247-250, 1997. 